\title{
Análisis de la expresión información local en siete documentos de apoyo al desarrollo de las bibliotecas públicas
}

\author{
José Luis Herrera Morillas \\ Margarita Pérez Pulido \\ Universidad de Extremadura (España)
}

\section{Resumen}

Se localiza, analiza y comenta la expresión información local en siete documentos sobre bibliotecas públicas: Manifiesto de la Unesco (1994), Declaración de Copenhague (1999), La biblioteca pública: un centro para la sociedad de la información (2000), Directrices IFLA/Unesco (2001); Pautas sobre los servicios de las bibliotecas públicas (2002), Pautas Pulman (2002) y Manifiesto de Oeiras (2003). Tras exponer los diferentes aspectos que encierra este concepto, se defiende que el papel de este tipo de información en la biblioteca pública actual está muy vinculado a la tendencia globalizadora de nuestra sociedad.

Palabras clave: Información local. Bibliotecas públicas.

\section{Abstract}

The expression local information in seven documents on the public libraries is located, analyzed and commented: Manifesto of UNESCO (1994); Declaration of Copenhagen (1999), La biblioteca pública: un centro para la sociedad de la información (2000), Guidelines IFLA/Unesco (2001), Pautas sobre los servicios de las bibliotecas públicas (2002); Pulman Guidelines (2002) and Manifesto of Oeiras (2003). After exposing the different aspects that this concept locks up, we defend that the roll of this kind of information in the present public library is very related to the tendency towards the globalization of our society.

Keywords: Local information. Public libraries.

\section{Introducción}

La importancia que se está dando a la información local en el ámbito de las bibliotecas públicas y las crecientes referencias a la misma en las publicaciones y eventos sobre estos centros nos han llevado realizar un estudio del concepto información local utilizando como fuente los documentos más recientes y de mayor difusión, de 
apoyo para el impulso y desarrollo de las bibliotecas públicas, que contienen referencias a este tipo de información. Este es el objetivo general del trabajo que presentamos. Se han analizado siete documentos que enumeramos a continuación, en orden cronológico de elaboración, aportando unas breves notas sobre su contenido.

\section{Manifiesto de la Unesco sobre la biblioteca pública, 1994}

Se trata de un manifiesto preparado por la Sección de Bibliotecas Públicas de la IFLA y aprobado por la Unesco en noviembre de 1994. Incluye una definición básica de la biblioteca pública, sus misiones y diversos apuntes sobre legislación, financiación y redes.

\section{Declaración de Copenhague "Algo para todos:} las bibliotecas públicas y la sociedad de la información. Congreso”, 1999

El 14 y el 15 de octubre de 1999 se reunieron en Copenhague responsables políticos y administrativos de 31 países europeos para analizar el papel esencial de las bibliotecas públicas en las emergentes sociedades de la información. Los asistentes a la reunión debatieron sobre los papeles clave que ya desempeñan muchas bibliotecas públicas al fomentar la identidad de la comunidad, el desarrollo económico, el aprendizaje continuo y la diversidad cultural. Al final de la reunión, los presentes aprobaron esta declaración como una manifestación de intenciones para el futuro de las bibliotecas públicas en el continente europeo.

\section{La biblioteca pública: un centro para la sociedad de la información, Subdirección General de Coordinación Bibliotecaria, 2000}

Se expone qué es y qué no es la biblioteca pública, los rasgos que la definen (universalidad, gratuidad y ámbito local), qué ofrece (funciones, servicios y recursos) y qué puede aportar a la sociedad de la información.

Directrices IFLA/Unesco para el desarrollo del servicio de bibliotecas públicas, FIAB/Unesco, 2001

Documento que contiene normas y recomendaciones para la prestación del servicio de biblioteca pública. Recoge aspectos como definición, colecciones, servicios, personal y cooperación. Incluye ejemplos de servicio de biblioteca pública de diversos ámbitos geográficos.

\section{Pautas sobre los servicios de las bibliotecas públicas, Ministerio de Educación y Cultura, 2002}

La iniciativa de elaborar estas Pautas surgió en el ámbito del programa de cooperación bibliotecaria entre el Ministerio de Educación, Cultura y Deporte y las comunidades autónomas. Su objetivo es facilitar a las instituciones y a los profesionales que trabajan en las bibliotecas públicas un instrumento que sirva de guía para la creación de bibliotecas públicas y para la planificación y prestación de los 
correspondientes servicios. Asimismo quieren ser un elemento básico que impulse el desarrollo de las bibliotecas públicas en toda España.

\section{Pautas Pulman (Public Libraries Mobilising Advanced Networks), 2002}

Elaboradas por los integrantes del proyecto Pulman, abordan los aspectos de los servicios y actividades más innovadoras, los nuevos servicios desarrollados en el entorno digital y el futuro de las bibliotecas públicas en la "Europa electrónica".

\section{Manifiesto de Oeiras, plan de trabajo de Pulman para e-Europe, 2003}

Aprobado por la Conferencia Europea sobre las Bibliotecas Públicas, los Museos y los Archivos (Oeiras, 13 y 14 marzo de 2003), subraya el papel de las bibliotecas públicas para satisfacer necesidades de los ciudadanos en la era digital. Impulsa la cooperación en el ámbito local con otras instituciones culturales, archivos y museos.

\section{Las alusiones a la información local: un recorrido por los textos}

El Manifiesto de la Unesco (1994) concibe la biblioteca pública como un centro local de información que facilita a sus usuarios todas las clases de conocimiento e información.

La Declaración de Copenhague (1999) incluye dentro de las funciones de la biblioteca pública el apoyo al crecimiento de las comunidades, ya que les proporciona servicios de información diseñados para atender las necesidades locales. Y entre sus misiones está prestar servicios adecuados de información a empresas, asociaciones y agrupaciones de ámbito local.

El documento La biblioteca pública: un centro para la sociedad de la información (2000), al explicar los servicios que ofrecen estos centros incluye un servicio centrado en la información local: dentro del servicio de información, la biblioteca pública, como entidad eminentemente local, debe procurar el mayor nivel de profundidad en lo que se refiere a la información local. Para ello, alberga una colección completa de materiales en todo tipo de soportes que contengan información relacionada con su localidad, y además establece y mantiene relaciones con todas las instituciones locales que puedan ser generadoras o consumidoras de información de interés local.

Enumera las ventajeas que puede aportar la biblioteca pública a la sociedad de la información, y una de ellas es la de contribuir a la preservación y difusión del patrimonio cultural de su comunidad, convirtiéndose en proveedora de información local en Internet.

Las Directrices IFLA/Unesco para el desarrollo del servicio de bibliotecas públicas (2001) indican como finalidad de la biblioteca pública la responsabilidad particular de recoger información local y de hacerla fácilmente accesible.

Scire. $13: 1$ (en.-jun. 2007) 121-132. ISSN 1135-3716. 
Otro aspecto relacionado con el papel y la finalidad de la biblioteca pública es la cultura local: la biblioteca pública debe ser una institución fundamental de la comunidad en la que se encuentra, en lo que se refiere al acopio, la preservación y la promoción de la cultura local en todas sus modalidades. Puede hacerlo de diferentes maneras: por ejemplo, manteniendo los fondos relativos a la historia del lugar, organizando exposiciones y narraciones orales, editando publicaciones de interés local o creando programas interactivos sobre temas locales. Cuando la tradición oral sea un importante método de comunicación, la biblioteca pública deberá fomentar su continuación y expansión.

$\mathrm{Al}$ definir el establecimiento de los fondos, entre los distintos recursos se indica que es vital desarrollar las fuentes de información local, y entre ellas los periódicos locales, regionales y nacionales y la documentación sobre la historia local.

En el programa de establecimiento de fondos de bibliotecas nuevas se expresa que en algunos países se utiliza material de centros nacionales o provinciales para complementar el fondo local.

Sobre los índices de adquisiciones y eliminación para las colecciones especiales se menciona que estos pueden no ser pertinentes para ciertas partes de los fondos, para colecciones concretas o cuando existan circunstancias peculiares. En tales casos, la política de los fondos ha de reflejar estas necesidades especiales. Entre dichas excepciones está la documentación sobre la historia local: se deberá recopilar y conservar materiales relacionados con la historia de la localidad y ponerlos a la disposición de los usuarios.

Para prestar servicios que correspondan a las necesidades de toda la comunidad, la biblioteca pública debe establecer el alcance de esas necesidades. Como las expectativas cambian, será preciso repetir periódicamente este proceso, tal vez cada cinco años. La evaluación de las necesidades de la comunidad es un proceso en el que la biblioteca acopia información detallada sobre la comunidad local y sus requerimientos en materia de información. La planificación y la elaboración de políticas se basan en los resultados de esta evaluación, lo que permite adecuar los servicios a las necesidades. En algunos países, la preparación y evaluación de las necesidades de la comunidad es un requisito reglamentario de la autoridad local. La información que se ha de recabar incluirá datos sociodemográficos de la comunidad local (por ejemplo, distribución por edades y por sexos, diversidad étnica y nivel de instrucción), datos sobre los organismos existentes en la comunidad (como establecimientos educativos, centros de salud, hospitales, establecimientos penitenciarios y agrupaciones de voluntarios) e información sobre las empresas y los comercios de la localidad.

Dentro del trabajo con los medios de comunicación para la comercialización y promoción se debe preparar al personal de la biblioteca para utilizar estos me- 
dios a fin de promover el servicio y de responder a sus consultas. El personal ha de estar en condiciones de redactar artículos para los periódicos locales y de preparar comunicados de prensa. Asimismo, tiene que estar familiarizado con las técnicas de la oratoria y de entrevistas por radio y televisión. También debe poder promover la biblioteca y sus servicios en las redes informáticas y de telecomunicaciones, incluida la creación de sitios sobre la biblioteca en la Red.

Las Pautas sobre los servicios de bibliotecas públicas (2002) defienden que la biblioteca pública se erige en el primer centro de información local, portal de acceso a la información que las tecnologías ponen a nuestro alcance, centro de actividades culturales de primer orden, espacio de identidad que estimula los valores de interculturalidad, solidaridad y participación, lugar de convivencia y encuentro. La biblioteca pública tiene una especial responsabilidad en lo relativo a la recopilación y el fácil acceso a la información local, para que se mantenga viva la historia de la comunidad a la que sirve y se desarrolle la cultura local.

En los servicios a los usuarios se recomienda un servicio de información local y comunitaria, centrado en la información básica sobre sanidad, empleo, vivienda, impuestos, protección legal, derechos políticos y sociales, consumo, educación, cultura, ocio..., que permita a los ciudadanos la integración y la participación efectivas en la sociedad. Información producida por la comunidad e información acerca de la comunidad, en especial la que refleje y difunda su identidad y su desarrollo cultural. La información comunitaria, ya sea elaborada por la propia biblioteca o por otros agentes, debe considerarse parte integrante de la colección o de los recursos de una biblioteca.

En cuanto al servicio de actividades culturales se aconseja la celebración de exposiciones bibliográficas, artísticas, científicas, divulgativas, etcétera, en las que se prestará una especial atención a los temas de interés local; actividades de fomento e impulso de la narración oral, a fin de preservar y difundir las culturas, la historia y tradiciones de la comunidad local; y otras actividades que impulsen las manifestaciones culturales locales.

En el capítulo que aborda los recursos de información, se señala que la colección debe entenderse como un bien de uso orientado al servicio, y no tanto como un bien inventariable y de conservación, salvo en lo que concierne al patrimonio bibliográfico y a la colección local. La biblioteca pública conservará durante tiempo indefinido los materiales de su sección de colección local, pero se limitará a conservar el resto de los fondos durante el periodo de tiempo en el que los materiales respondan a criterios de calidad y actualidad propios de la lectura pública y a los objetivos de la biblioteca.

En la gestión de la colección hay que plantearse configurar determinadas secciones debido a circunstancias o necesidades específicas. Se trataría de la creación, Scire. $13: 1$ (en.-jun. 2007) 121-132. ISSN 1135-3716. 
mantenimiento, difusión y conservación de colecciones como las relacionadas con la cultura y la historia locales.

En la introducción de las Pautas Pulman (2002) se menciona la encrucijada en la que se encuentran la bibliotecas públicas en Europa en la actualidad y se precisa que ante esta situación existe una evidente necesidad de desbloquear, revelar y hacer más accesibles los contenidos digitales de ámbito local que en la actualidad albergan archivos y museos, los cuales pueden aportar un sentimiento de comunidad en un mundo cada vez más globalizado.

En la pauta "Participación ciudadana en nuevas formas de gobierno cívico" se indica — en cuanto a las áreas de actuación— que las bibliotecas públicas siempre han abogado por la libertad de pensamiento y el derecho al libre acceso a la información. Ya han sobrepasado ese papel tradicional. El concepto de biblioteca como centro al servicio de la comunidad y como depósito de la memoria local es, hoy día, una realidad en muchos países. Como instituciones clave en el acceso a la información, las bibliotecas pueden y deben capitalizar los programas cooperativos sociales dentro del ámbito local para desarrollar y ofrecer nuevos servicios basados en las tecnologías de la sociedad de la información (TSI) en apoyo de la participación democrática. Entre las pautas de buena práctica se hace referencia a que las bibliotecas públicas deben desarrollar su posición actual para actuar como puntos de acceso a la administración en línea y para ejercer el derecho al voto a través de Internet, proporcionando guía y asesoramiento; fomentar la participación ciudadana en las nuevas formas de gobierno por medio de programas informativos, servicios informáticos interactivos y charlas coloquio; proporcionar acceso a la información de la administración local y central, sus planes y proyectos; preparar a los miembros de la comunidad a la que sirven, pidiéndoles consulta, difundiendo información de calidad y formándoles en las nuevas tecnologías, para que contribuyan en la toma de decisiones.

Las bibliotecas públicas son una institución clave en la participación ciudadana debido a variadas razones; una de ellas: dar acceso a la información de las administraciones local y central, sus proyectos y programas.

La pauta "Servicios de biblioteca pública para niños y centros de enseñanza" recoge como buena práctica proporcionar acceso y estructurar adecuadamente contenidos educativos, de óptima calidad, apropiados y atractivos, que puedan utilizarse dentro de los nuevos entornos de aprendizaje basados en las TSI. Una fuente de potencial importancia para estos contenidos es el material depositado en las bibliotecas, museos y archivos, que puede servir de apoyo a los planes de estudios de historia local, arte y medio ambiente.

En la pauta "Acceso a contenidos culturales diversos e información a la comunidad" se destaca — dentro de las áreas de actuación— que los museos, los ar- 
chivos y otras instituciones del patrimonio están digitalizando sus fondos a un buen ritmo; esa digitalización en continuo aumento presta especial atención a la información de interés local. También se señala que las bibliotecas públicas deben trabajar en conjunto con otras instituciones de la memoria y el patrimonio, como son los museos y archivos, para crear nuevos recursos que incluyan temas de historia local, actividades culturales, contenidos culturales locales e información local. Los países más avanzados en este campo son los del norte de Europa, especialmente Escandinavia. En los Estados Unidos, Irlanda y el Reino Unido, el interés por la historia familiar y la genealogía obedece a su larga tradición migratoria. En la actualidad se están incrementando las labores de digitalización en las instituciones del patrimonio cultural ubicadas en la localidad. El acceso a la información comunitaria y cultural de la localidad no está bien cubierto por otros portales o buscadores generales. Es aquí donde las bibliotecas pueden aportar una contribución inestimable.

Como pauta de buenas prácticas se sitúa el acceso a la información comunitaria y cultural de la localidad, que es un área poco cubierta por otros portales o buscadores generales. Las bibliotecas pueden realizar una contribución fundamental en dicho campo.

De cara al futuro, la digitalización de las colecciones culturales continuará hasta que una amplia proporción del patrimonio cultural europeo esté disponible de una manera u otra en formato digital. Los contenidos de interés local formarán una parte cada vez más importante de este panorama. Los museos, archivos y bibliotecas públicas tendrán que trabajar en conjunto con asociaciones de voluntarios y vecinales para asegurar que este material esté disponible.

De la pauta "Desarrollo de sistemas integrados de gestión de bibliotecas" hay que destacar que se indica como buena práctica el que los avances en el campo de los SIGB se relacionen con el desarrollo de otras funcionalidades que vayan más allá de los módulos básicos o tradicionales, tales como gestión de información local. En la actualidad son muchas las bibliotecas que requieren y precisan módulos adicionales como el de gestión de información local en formato digital.

Finalmente, la pauta "Localización, recuperación y descripción de recursos" menciona como buena práctica que algunas bibliotecas comienzan a crear sus propios portales y gateways, por ejemplo, para apoyar clumps, servicios de información local, y para proporcionar apoyo al aprendizaje. Así, por ejemplo, la corporación municipal de Essex, en el Reino Unido, ha sido la pionera en desarrollar un sistema de información a la comunidad descentralizado. En lugar de las tradicionales bases de datos centralizadas de información local, Essex Online (que nació a raíz del proyecto Seamless), utiliza el protocolo Z39.50 y Harvest para buscar en las bases de datos y servidores web de las organizaciones participantes mediante una sola búsqueda integrada. A esto también se le denomina integración en Scire. $13: 1$ (en.-jun. 2007) 121-132. ISSN 1135-3716. 
profundidad. En la actualidad más de treinta organizaciones de ámbito local (ayuntamientos, facultades y escuelas universitarias, departamentos comerciales, autoridades sanitarias, empresas de servicios, periódicos locales, agencias de voluntariado, etcétera) incorporan su información dentro del sistema, y su número va en aumento.

Para que el sistema pueda realizar búsquedas a través de estos conjuntos de datos diseminados, los miembros utilizan un perfil común de metadatos basado en el e-gms, que a su vez se basa en el Dublin Core, y un tesauro en común para la asignación de los descriptores a los temas. Recientemente, el New Opportunities Fund ha concedido financiación al condado de Essex para desarrollar el sistema a nivel nacional introduciendo datos de los más importantes distribuidores de información del país, como por ejemplo nhsDirect Online, y poniendo estos mecanismos de búsqueda a disposición de ocho autoridades locales más, hasta abarcar un total de 6 millones de personas.

En el Manifiesto de Oeiras (2003), entre las acciones a las que se invita a los ministros, responsables políticos y profesionales en los ámbitos nacional y local están desarrollar una colaboración eficaz entre el sector del patrimonio cultural local y otros sectores económicos y sociales clave (por ejemplo, educación, empleo, turismo, organizaciones comunitarias, etcétera) para facilitar la reorganización de los servicios locales, así como su provisión y gestión rentable; y proporcionar acceso interactivo a los contenidos por medio de estados de la cuestión, recursos digitales multimedia que documenten la historia local, la literatura, el arte, la música y los intereses de la comunidad, presentados cuando sea conveniente como recursos de aprendizaje.

\section{Reflexiones y conclusiones}

Tras la lectura de estos documentos y en relación con la expresión información local se pueden distinguir las siguientes funciones de la biblioteca pública: $a$ ) centro de información de carácter enciclopédico para la localidad, con especial atención a los temas de actualidad; $b$ ) centro que recopila información sobre la localidad y que organiza y apoya todo tipo de iniciativas y actividades que potencien la cultura local; $c$ ) centro que diseña y realiza recursos para la preservación y difusión digital del patrimonio cultural local.

Estas funciones se han ido perfilando de forma progresiva. En un primer momento las alusiones a la información local se centran en el primer aspecto: la biblioteca como centro de información, es decir, como centro al que los ciudadanos acuden para resolver sus necesidades de información. La biblioteca pública ha de convertirse en un lugar donde el ciudadano pueda recabar todo tipo de información; debe ser el referente para que cualquier persona de la localidad pueda satisfacer sus necesidades de información. De este modo pasa a ser una unidad de información 
de ámbito local capaz de ofrecer al ciudadano la información y/o documentación necesaria para la realización de actividades relacionadas con la cultura, la formación, el trabajo, el ocio, el ejercicio de deberes sociopolíticos y la ejecución de cualquier trámite administrativo (Colomer y Silleras, 1998). En los dos primeros documentos analizados (Manifiesto de la Unesco y Declaración de Copenhague) las breves menciones a la información local se hacen bajo esta concepción.

A continuación se va a subrayar también la importancia de que la biblioteca sea el mejor centro especializado en información sobre la localidad, proveedor de información local, facilitando así la información que la comunidad necesita en relación con su localidad. En este sentido debe participar de forma activa en el apoyo de la cultura local, en la difusión del conocimiento de la localidad, asumiendo el papel de promotor y divulgador de la cultura local, etcétera (1). Esta función se desprende de la concepción de la biblioteca como una institución social, y por ello ha de estar comprometida con la promoción de las manifestaciones culturales de las comunidades en las que trabaja (Betancur, 2002). El resto de los documentos analizados tratan ampliamente esta dimensión de la información local. Entre ellos destacamos las alusiones de las Directrices IFLA/Unesco para el desarrollo del servicio de bibliotecas públicas.

La biblioteca pública local se va configurando de este modo como un centro especializado en información local, mejor que otros tipos de bibliotecas (autonómicas, nacionales, etcétera) que, por tener unas funciones y tareas a veces demasiado ambiciosas, no están en condiciones de recopilar y conservar con exhaustividad toda la información y la documentación relacionada con una determinada localidad. Esta realidad se manifiesta de modo muy evidente en el caso de los materiales menores (folletos, catálogos, carteles, tarjetas, etcétera), pues una biblioteca nacional, e incluso una autonómica, se puede ver fácilmente desbordada a la hora de controlar estos documentos. La exhaustividad y la mayor cobertura de las colecciones y los recursos relacionados con la localidad son las que realmente pueden dar una seña de identidad propia a las bibliotecas públicas de ámbito local frente a otros tipos de bibliotecas (Fornas, 2002, pp. 56-57).

Finalmente, con el desarrollo de la sociedad de la información y la aplicación e impacto de las nuevas tecnologías en el ámbito bibliotecario — sin abandonar las alusiones a los otros aspectos, a los que se sigue dando gran importancia al redefinirlos en el nuevo contexto tecnológico-, el acento se pone en la realización de iniciativas que supongan la aplicación de estas tecnologías al servicio de la elaboración de recursos con contenidos sobre la localidad y sobre el patrimonio bibliográfico de la biblioteca, especialmente utilizando Internet como apoyo de la biblioteca en tanto que centro preservador y difusor del patrimonio cultural. Así, las bibliotecas deben integrar esta información propia a los sistemas de información globalizados (2). Los dos últimos documentos seleccionados, más recientes

Scire. $13: 1$ (en.-jun. 2007) 121-132. ISSN 1135-3716. 
en el tiempo (Pautas Pulman y Manifiesto de Oeiras), abordan esta última función; con mayor amplitud las Pautas Pulman, dedicadas íntegramente al desarrollo de los nuevos servicios de las bibliotecas públicas en el entorno digital.

También hay que hacer notar el interés de los gobiernos y organismos nacionales y/o supranacionales en apoyar esta corriente, el cual se refleja en las recientes recomendaciones y normativas promulgadas, que implican o recogen medidas relacionadas con la difusión del patrimonio cultural, especialmente en el ámbito digital. Responden a recomendaciones, principios o informes que pretenden impulsar y orientar proyectos relacionados con la difusión a través de la digitalización de este patrimonio (3).

Por lo tanto, la difusión digital del patrimonio documental y cultural se presenta en el entorno de la biblioteca pública como una actividad en auge y muy recomendada. De todos modos, no se puede ignorar que la puesta en marcha de estos proyectos en un entorno digital está condicionada por una serie de factores que se deben conocer, y hay que estar al día de sus últimas tendencias. Se trata de factores de carácter normativo, técnico, jurídico, financiero y político que toda persona encargada de la planificación de un proyecto de estas características ha de tener en cuenta antes de decidir su inicio (Bequet, 2000).

A nuestro entender, en la globalización está la clave de la importancia que se le está dando a la información local en relación con la biblioteca pública y, por tanto, del crecimiento progresivo de las alusiones a este concepto constatadas en los textos estudiados. La posibilidad de intercambiar información instantáneamente y a escala mundial ha provocado el debilitamiento de las fronteras y el contacto entre los pueblos. Esta es la tendencia de la globalización. En la sociedad de hoy, que persigue la optimización de los sistemas productivos, se experimenta un proceso de uniformización que pone en peligro las libertades personales y las mismas culturas nacionales y locales. Se debe reaccionar defendiendo y apoyando todo lo que respete y aliente el papel de los individuos y los grupos, tener en cuenta las individualidades culturales y sociales. En este sentido afirma Betancur (2002, p. 41):

Las localidades se han convertido en escenarios de una desgarradora tensión entre las presiones globalizadoras del capital internacional y la necesidad de responder a las demandas internas de una población que padece los efectos de la política de ajuste estructural, de la apertura económica y de múltiples formas de exclusión social y política... Uno de los retos es la creación de servicios de información local, para que recojan, organicen y difundan de manera intencional y sistemática la información generada por la comunidad radio de acción de la biblioteca pública, en diferentes aspectos de su desarrollo, utilizando diversos medios, formatos y soportes para la transferencia de la información y en función de los procesos de fortalecimiento de las identidades culturales y la participación ciudadana y comunitaria. 


\section{Notas}

(1) Esta idea está desarrollada ampliamente en el trabajo Bibliotecas públicas municipales: el derecho de todos a acceder a la cultura. Sevilla: Defensor del Pueblo Andaluz, 2000.

(2) Como ejemplo de esta tendencia citamos el Proyecto Jumilla, llevado a cabo por la Biblioteca Municipal de esta localidad murciana y concebido como un sistema integrado de información local especializado en proporcionar a los ciudadanos información y documentación cultural, turística y comunitaria. Para más información sobre el mismo véase González Olivares, J. L. Proyecto Jumilla: desarrollo de un sistema integrado de información local. // Actas de las VI Jornadas Españolas de Documentación: Los sistemas de información al servicio de la sociedad. Valencia: Fesabid, 1998. http://fesabid98.florida-uni.es/Comunicaciones/jl_gonzalez/j1_gonzalez.htm (200409-05).

(3) Por ejemplo, las Recomendaciones de la Conferencia Internacional sobre Digitalización del Patrimonio Cultural Europeo (1999). // Correo Bibliotecario. 40 (mar. 2000) (sección amplio alcance). http://www.bcl.jcyl.es/correo/Correo40/index.html (2004-0905); los Principios de Lund (Suecia, 4 de abril de 2001). http://www.cordis.lu/ist/ directorate_e/digicult/lund_p_browse.htm (2005-09-24); Memoria del mundo: directrices (ed. revisada, 2002), preparada por Ray Edmondson. París: Unesco, 2002; OryLavollée, B. La diffusion numérique du patrimoine, dimension de la politique culturelle: rapport à Mme. la Ministre de la Culture et de la Communication. París: Ministère de la Culture et de la Communication, 2002.

\section{Referencias}

Bequet, G. (2000). Numérisation et patrimoine documentaire. // Bulletin des Bibliothèques de France. $45: 4$ (2000) 67-72.

Betancur, A. M. (2002). Un lugar en el mundo: los servicios de información local en la biblioteca pública. // Métodos de Información. $51: 9$ (jul. 2002) 38-43.

La biblioteca pública: un centro para la sociedad de la información (2000). Subdirección General de Coordinación Bibliotecaria. http://travesia.mcu.es/documentos/bp-def.asp (2005-06-10).

Colomer Bartrolí, M.; Silleras, M. E. (1998). Tecnología y calidad al servicio del ciudadano: el centro de información municipal. // Actas de las VI Jornadas Españolas de Documentación: Los sistemas de información al servicio de la sociedad. Valencia: Fesabid, 1998. http://fesabid98.florida-uni.es/Comunicaciones/me_silleras.htm (2004-09-05).

Declaración de Copenhague: Algo para todos: las bibliotecas públicas y la sociedad de la información. Congreso (1999). http://travesia.mcu.es/documentos/Copenhague.pdf (2004-08-07).

Directrices IFLA/Unesco para el desarrollo de servicios de bibliotecas públicas (2001). http://unesdoc.unesco.org/images/0012/001246/124654s.pdf (2004-09-05).

Fornas Carrasco, R. (2002). La biblioteca pública municipal y los servicios de información local. // Métodos de Información. 51:9 (jul. 2002) 56-57.

Scire. $13: 1$ (en.-jun. 2007) 121-132. ISSN 1135-3716. 
Manifiesto de Oeiras, plan de trabajo de Pulman para e-Europe (2003). http://travesia.mcu.es/ documentos/Oeiras.pdf (2005-10-03).

Manifiesto de la Unesco sobre la biblioteca pública (1994). http://www.fundaciongsr.es/ documentos/manifiestos/mani94es.pdf (2004-09-05).

Pautas Pulman (2002). Trad. esp. de Nassimah Rose Reynolds Palis y Antonio Agustín Gómez Gómez. http://www.cult.gva.es/DGLB/images/pautaspulman.pdf (2004-09-05).

Pautas sobre los servicios de las bibliotecas públicas (2002). Madrid: Ministerio de Educación Cultura y Deporte, 2002. 